\title{
LSTrAP-Crowd: prediction of novel components of bacterial ribosomes with crowd-sourced analysis of RNA sequencing data
}

Benedict Hew, Qiao Wen Tan, William Goh, Jonathan Wei Xiong Ng and Marek Mutwil (D)

\begin{abstract}
Background: Bacterial resistance to antibiotics is a growing health problem that is projected to cause more deaths than cancer by 2050. Consequently, novel antibiotics are urgently needed. Since more than half of the available antibiotics target the structurally conserved bacterial ribosomes, factors involved in protein synthesis are thus prime targets for the development of novel antibiotics. However, experimental identification of these potential antibiotic target proteins can be labor-intensive and challenging, as these proteins are likely to be poorly characterized and specific to few bacteria. Here, we use a bioinformatics approach to identify novel components of protein synthesis.

Results: In order to identify these novel proteins, we established a Large-Scale Transcriptomic Analysis Pipeline in Crowd (LSTrAP-Crowd), where 285 individuals processed 26 terabytes of RNA-sequencing data of the 17 most notorious bacterial pathogens. In total, the crowd processed 26,269 RNA-seq experiments and used the data to construct gene co-expression networks, which were used to identify more than a hundred uncharacterized genes that were transcriptionally associated with protein synthesis. We provide the identity of these genes together with the processed gene expression data.

Conclusions: We identified genes related to protein synthesis in common bacterial pathogens and thus provide a resource of potential antibiotic development targets for experimental validation. The data can be used to explore additional vulnerabilities of bacteria, while our approach demonstrates how the processing of gene expression data can be easily crowd-sourced.
\end{abstract}

Keywords: Co-expression, Bacteria, Ribosome, Protein synthesis, RNA-seq, Crowdsourcing

\section{Background}

Bacterial resistance to antibiotics is a serious and growing concern in public health, taking ca. 99,000 lives and costing 21-34 billion USD per year in the USA [1]. Methicillin-resistant Gram-positive Staphylococcus aureus (MRSA) and Gram-negative Pseudomonas aeruginosa are the leading causes of serious infections as they

\footnotetext{
* Correspondence: mutwil@ntu.edu.sg

School of Biological Sciences, Nanyang Technological University, 60 Nanyang Drive, Singapore 637551, Singapore
}

form biofilms. Biofilms are complex bacterial communities embedded in an extracellular matrix, and these communities are able to resist antimicrobial agents [2]. For instance, bacteria can be up to $1000 \times$ more tolerant to antibiotics when they grow as a biofilm, compared to single-cell suspension (planktonic cells). Consequently, new antibiotics are urgently needed to combat these resistance mechanisms, either alone or in combination with existing drugs.

(c) The Author(s). 2020 Open Access This article is licensed under a Creative Commons Attribution 4.0 International License, which permits use, sharing, adaptation, distribution and reproduction in any medium or format, as long as you give appropriate credit to the original author(s) and the source, provide a link to the Creative Commons licence, and indicate if changes were made. The images or other third party material in this article are included in the article's Creative Commons licence, unless indicated otherwise in a credit line to the material. If material is not included in the article's Creative Commons licence and your intended use is not permitted by statutory regulation or exceeds the permitted use, you will need to obtain permission directly from the copyright holder. To view a copy of this licence, visit http://creativecommons.org/licenses/by/4.0/ The Creative Commons Public Domain Dedication waiver (http://creativecommons.org/publicdomain/zero/1.0/) applies to the data made available in this article, unless otherwise stated in a credit line to the data. 
More than half of the antibiotics currently in use target the bacterial ribosome, typically at the elongation step of protein synthesis [3], through direct or proximal binding of the peptidyl transferase center (PTC) which catalyzes peptide bond formation [4]. PTC-targeting antibiotics (e.g., lincosamides, pleuromutilins, chloramphenicol, and group A streptogramins), inhibit protein synthesis by obstructing the proper positioning of the tRNA substrates [5].

Bacteria can be intrinsically less sensitive to antibiotics due to less efficient uptake of antibiotics or mutations in ribosomal proteins that result in decreased drug-binding efficiency $[3,6]$. The most frequently encountered acquired resistance mechanism involves the methylation of the ribosomal RNA (e.g., by Erm family methyltransferases), which results in decreased drug-binding efficiency and increased viability in the presence of antibiotics [7, 8]. As modification of the ribosomes can result in a decrease in fitness, these methyltransferases genes tend to be induced by the relevant antibiotics through translation attenuation $[9,10]$. Alternatively, the antibiotics can also be modified, pumped out, or degraded, thus lowering the intracellular concentration to non-toxic levels $[3,11]$. Another mechanism is ribosome protection, where the antibiotic is actively dislodged from the ribosome by ATP-binding cassette $\mathrm{F}(\mathrm{ABC}-\mathrm{F})$ protein, as observed in many clinical isolates (e.g., Pseudomonas aeruginosa, Escherichia coli, Staphylococcus aureus, Enterococcus faecalis and Listeria monocytogenes) [12-14].

While the structure of the ribosomes is well conserved, structural features of ribosomes may vary significantly between different species, suggesting species-specific adaptations of protein synthesis [15-23]. For example, structural analysis of mycobacterial ribosome revealed that the 30S ribosomal subunit lacks the protein bS21 that is found in Escherichia coli. Instead, the mycobacteria employ a unique protein bS22 near the decoding center (DC), thereby keeping the overall number of ribosomal proteins in $30 \mathrm{~S}$ subunit the same as in E. coli [19]. Thus, the identification of novel bacteria ribosomal components has great potential for the development of species-specific antibiotics. However, the identification of these novel components using traditional molecular or structural biology approaches is time-consuming.

Bioinformatic approaches are used to predict gene function and can be used to identify novel components of protein synthesis. Newly sequenced genomes of all organisms are typically first annotated using sequence similarity analysis, where the genes are annotated based on the DNA/ protein sequence similarity to characterized genes/proteins [24]. While sequence similarity analysis is well established and gives a quick overview of gene functions in a new genome, it has its caveats as genes can (i) have multiple functions, (ii) sub- or neo-functionalize, and/or (iii) have no sequence similarity to characterized genes. Thus, while sequence similarity analysis is a powerful method, it requires other methods to complement it [24,25].

The wide availability of RNA sequencing (RNA-seq) data makes it possible to study gene function from the perspective of gene expression [24, 26-28]. Co-expression analysis is based on the observation that genes that have similar expression profiles across experiments tend to be functionally related [24, 25, 29]. These co-expressed genes can be identified by analyzing publicly available microarrays or RNA-seq data, and the co-expression relationships can be represented as networks. In a co-expression network, genes are represented as nodes, where edges connect co-expressed nodes (links) [30-40]. The networks can be mined for groups of highly connected genes (called clusters or modules) that likely represent genes that are involved in the same biological process. Due to the ubiquity of expression data, and the ability to complement DNA/protein sequence-based gene function prediction approaches, coexpression networks have become a popular tool to elucidate the function of genes. The networks have predicted the function of genes involved in a wide range of processes, such as various cellular processes [38, 41-43], transcriptional regulation [44], physiological responses to the environment and stress [45, 46], and the biosynthesis of metabolites [34, 47-49].

The amount of gene expression data has expanded vastly over the last decade, resulting in $>1000$-fold increase in nucleotide bases on NCBI Sequence Read Archive (SRA), from 11 TB (2010) to 12 PB (2020). Due to limitations in software used to estimate gene expression from RNA-seq data, analyzing all this data would have been unthinkable a decade ago. However, drastic improvements to the speed and efficiency of software, such as Kallisto [50] and salmon [51], allow the analysis of gigabytes of data on even a Raspberry Pi-like miniature computer [48]. Recently, combined the availability of cloud computing and the user-friendliness of the Jupyter notebooks to implement a large-scale transcriptomic analysis pipeline, LSTrAP-Cloud [47]. Importantly, though Google Colab, the pipeline gives access to a free cloud computer with 2 Xeon cores, with at least 15 GB of permanent storage (as provided by users Google drive account) and $12 \mathrm{~GB}$ of RAM, giving biologists both the software and hardware to perform large-scale co-expression analysis.

In this study, we introduce Large-Scale Transcriptomic Analysis Pipeline in Crowd (LSTrAP-Crowd). This simple pipeline was used by 285 undergraduate students to process RNA-seq data of some of the 17 most notorious bacterial pathogens. Within a week, the students processed 26,269 RNA-seq samples, comprising 263,757,103, 900 ( 263 billion) reads and 26.38 terabytes of data. The gene expression data was used to construct coexpression networks, which were mined for the presence of uncharacterized genes that were co-expressed with 
the bacterial ribosomes. In total, we have predicted more than 100 putative proteins to be involved in protein synthesis in the 17 bacterial pathogens.

\section{Results}

Obtaining and quality-controlling gene expression data for 17 bacterial pathogens

In this study, we analyzed the gene expression data of 17 notorious bacterial pathogens that cause numerous diseases, such as pharyngitis, tonsillitis, scarlet fever, cellulitis, erysipelas, rheumatic fever, post-streptococcal glomerulonephritis, necrotizing fasciitis, and many others (Table 1). While more bacterial pathogens were considered, we only analyzed bacteria that had at least 100 RNA-seq samples based on Illumina technology found in the Sequence Read Archive [52]. In total, 26,269 RNA-seq samples were analyzed.

The RNA-seq data was streamed by using a modified LSTRaP-Cloud pipeline (Fig. 1a), which gives each user a free Google Colab notebook equipped with a 2 core Xeon CPU and $12 \mathrm{~Gb}$ of RAM [47]. The modified pipeline, LSTrAP-Crowd, thus allows a large group of people to download the gene expression data collaboratively. Two hundred eighty-five first-year undergraduate students were divided into 60 groups, with each group tasked to download a maximum of 600 RNA-seq samples (Additional file 1: Table S1). The size of each RNA-seq sample was capped at $\sim 1 \mathrm{~Gb}$, allowing a person running the modified LSTRaP-Crowd pipeline to download $\sim 300$ RNA-seq samples per day [47].
Theoretically, $85,500 \quad(300 \times 285) \quad$ RNA-seq samples equivalent to $\sim 85 \mathrm{~Tb}$ could be processed per day by the classroom.

For each species, all the processed RNA-seq experiments were visualized as scatter plots that show the percentage $(y$-axis) against the number ( $x$-label) of reads pseudoaligned to the respective species' CDS (Fig. 1b). For each experiment, high pseudoalignment percentage indicates high sequence similarity to the CDS, whereas a high absolute number of reads indicates whether the experiment has sufficient data for meaningful coexpression analysis. In this study, a minimum threshold of 1 million reads pseudoaligned was required for the experiment to be considered. We removed samples with $\mathrm{n} \_$pseudoaligned $<1,000,000$ and with $\mathrm{p}$ _pseudoaligned values that were lower than the majority of the high p_pseudoaligned samples (typically $>30 \%$ ) (Fig. 2a, Additional file 2: Figure S1). The scatterplot pattern was different for each bacteria, most likely due to each bacteria having a different ratio of coding to non-coding DNA (Additional file 2: Figure S1). Samples that passed these thresholds (Fig. 2b) were used to build expression matrices (Additional file 3: Table S2, Additional file 4: Table S3, Additional file 5: Table S4, Additional file 6: Table S5, Additional file 7: Table S6, Additional file 8: Table S7, Additional file 9: Table S8, Additional file 10: Table S9, Additional file 11: Table S10, Additional file 12: Table S11, Additional file 13: Table S12, Additional file 14: Table S13, Additional file 15: Table S14, Additional file 16: Table S15, Additional file 17: Table S16, Additional file 18: Table S17, Additional file 19: Table

Table 1 Genomic properties of the 17 bacteria and the RNA-seq sample statistics

\begin{tabular}{|c|c|c|c|c|}
\hline Bacteria & Number of genes & Genome size (Mb) & Student groups analyzing the data & RNA-seq samples: passed QC/total \\
\hline Campylobacter jejuni & 1635 & 1.65 & 1 & $219 / 320$ \\
\hline Clostridioides difficile & 3769 & 4.27 & 1 & $182 / 381$ \\
\hline Enterococcus faecalis & 2579 & 3.11 & 1 & $81 / 195$ \\
\hline Escherichia coli & 4141 & 5.44 & 13 & $2494 / 7154$ \\
\hline Haemophilus influenzae & 2098 & 1.79 & 2 & $189 / 448$ \\
\hline Helicobacter pylori & 1720 & 1.62 & 1 & $84 / 167$ \\
\hline Klebsiella pneumoniae & 5141 & 5.78 & 2 & $536 / 639$ \\
\hline Listeria monocytogenes & 2817 & 2.78 & 2 & $370 / 572$ \\
\hline Mycobacterium tuberculosis & 4023 & 4.33 & 11 & $2414 / 6495$ \\
\hline Mycoplasma pneumoniae & 629 & 0.82 & 3 & $365 / 956$ \\
\hline Neisseria gonorrhoeae & 2159 & 2.15 & 1 & $102 / 371$ \\
\hline Pseudomonas aeruginosa & 6512 & 6.09 & 7 & $1372 / 2662$ \\
\hline Salmonella enterica & 4554 & 4.79 & 3 & $611 / 1284$ \\
\hline Staphylococcus aureus & 2638 & 2.90 & 5 & $1112 / 1963$ \\
\hline Streptococcus pneumoniae & 2043 & 2.13 & 2 & $422 / 647$ \\
\hline Streptococcus pyogenes & 1660 & 1.85 & 4 & 1340/1705 \\
\hline Vibrio cholerae & 3648 & 4.00 & 1 & $167 / 311$ \\
\hline
\end{tabular}




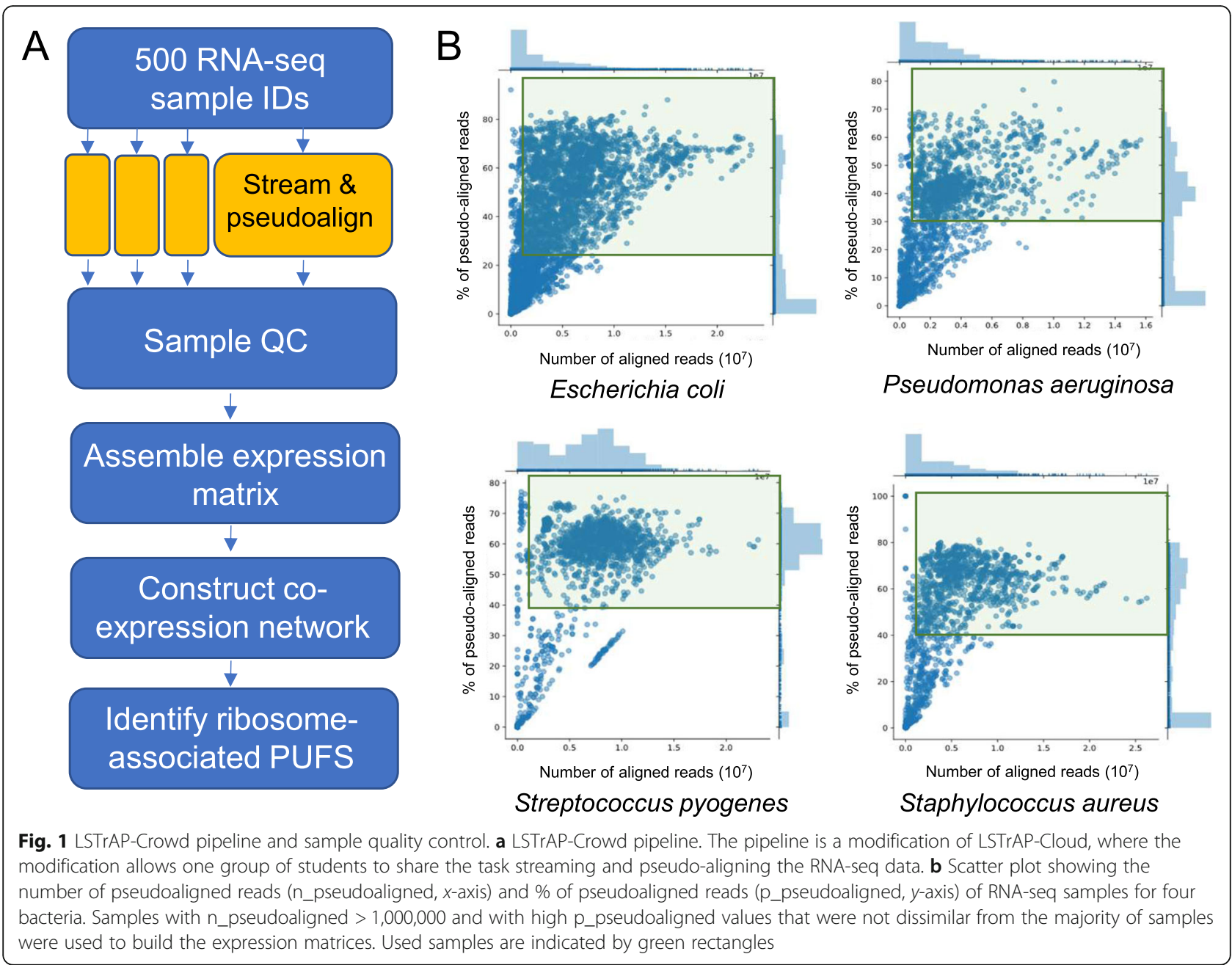

S18) and used for the co-expression analysis and identification of novel genes involved in protein synthesis.

\section{Construction and evaluation of co-expression networks for the 17 bacteria}

A small portion of real-world networks is scale-free [53], including co-expression networks (Mutwil et al., 2010). In scale-free networks, only a few genes are connected (correlated) to many genes, while the majority of genes show only a few connections [54]. Scale-free topology is hypothesized to ensure that the network remains mostly unaltered in case of mutations, and is an evolved property that ensures robustness against perturbations [55]. To demonstrate that the expression data of the 17 bacteria can generate biologically meaningful co-expression networks, we investigated whether the data can produce a typical scale-free network. All of the co-expression networks of the 17 bacteria showed a pattern indicative of scale-free topology, as plotting the number of connections a gene has (node degree) against the frequency of this association produced a negative slope (Fig. 3a). This confirms the scale-free topology of the co-expression networks and suggests that the networks are biologically relevant.

Interestingly, we observed that the power-law plots of some bacteria contain more nodes with a higher degree than expected from a network following power law (Fig. 3a, indicated by red squares). While the basis of this phenomenon is outside of the scope of this publication, we speculate that this is caused by the operon structure of the bacterial genes. Interestingly, certain bacteria, such as Vibrio cholerae (Fig. 3a) did not show this pattern (see Additional file 20: Fig. S2 for power law plots for all bacteria). Finally, Mycoplasma pneumoniae power-law plot showed a small number of points, indicating that few genes show PCC $>0.7$ in this bacteria. This could be attributed to most samples in this bacterium showing worse mapping statistics than the other 16 bacteria (Fig. 2a, Additional file 1: Fig. S1), indicating that perhaps the available CDS for Mycoplasma are of poor quality. 

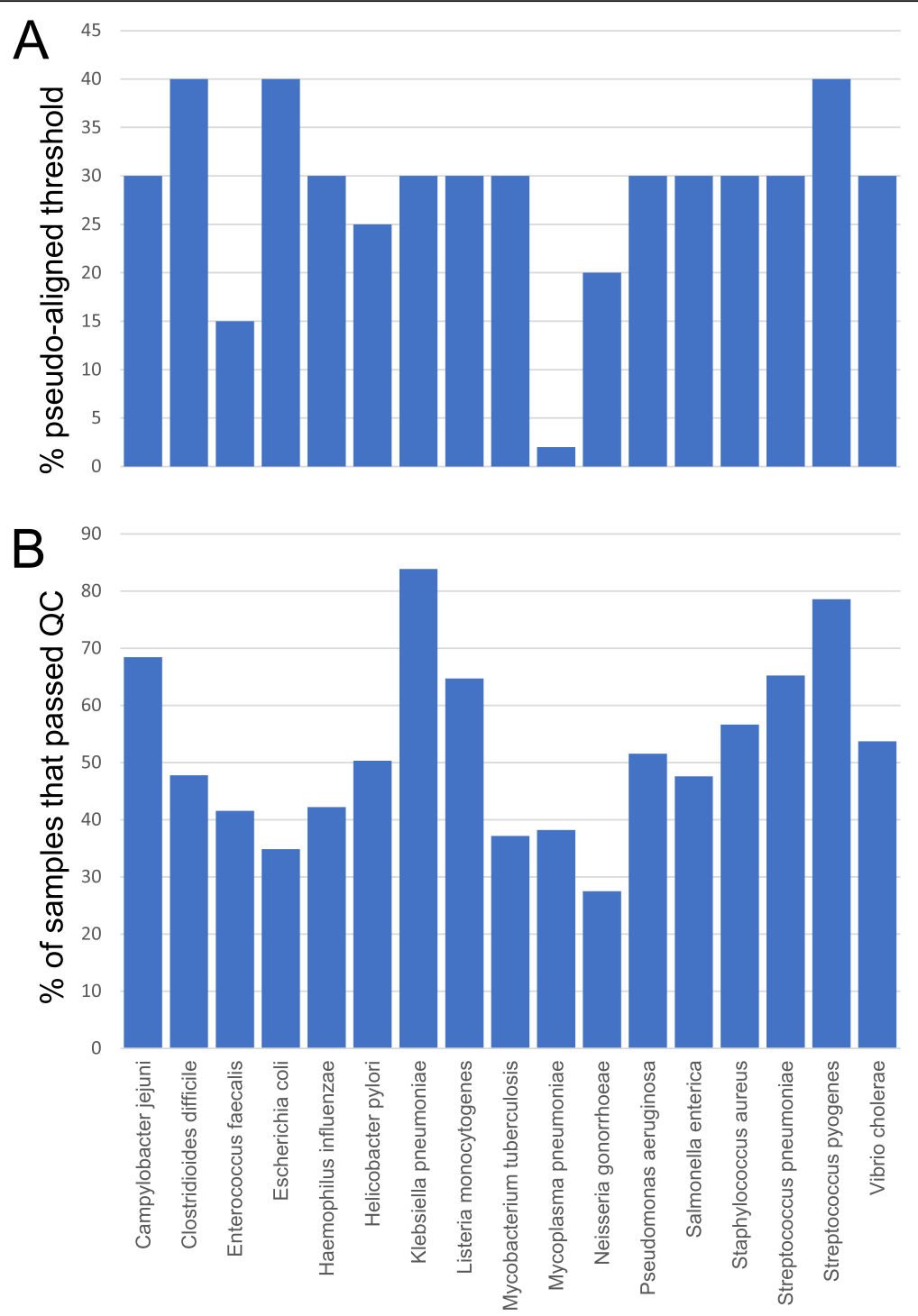

Fig. 2 Quality control of the 26,270 RNA-seq samples. a \% pseudoaligned (p_pseudoaligned) threshold set for the 17 bacteria. b The percentage of samples that passed (blue) or failed the p_pseudoaligned and n_pseudoaligned $>1,000,000$ thresholds

To demonstrate that our co-expression networks can be used to predict novel components of ribosomes, we investigated the co-expression neighborhood of AEA92696, a 30S ribosomal protein S18 from Enterococcus faecalis. The neighborhood was constructed by retrieving the top 50 genes with the highest PCC values to AEA92696 (Additional file 21: Table S19), where gene pairs with PCC $>0.7$ are connected (Fig. 3b). Out of 50 genes, $22(n=44 \%)$ were annotated as a component of the 30 S (e.g., S15, S4, S3) or 50S (e.g., L15, L3, L14) ribosomal subunit, indicating that genes in this neighborhood are involved in protein synthesis. Interestingly, 7 genes in the neighborhood are annotated as "hypothetical proteins" (Fig. 3b). Since these genes are found in the neighborhood that is likely to be involved in protein synthesis, we propose that these hypothetical proteins are also involved in protein synthesis in Enterococcus faecalis. We observed that ribosomal proteins (RP) show distinctively higher PCC values to other RPs in nearly all bacteria (Fig. 3c, RP-RP), when compared to PCC values between ribosomal proteins and non-ribosomal genes (Fig. 3c, RP-Other). Thus, ribosomal proteins tend to be neighbors to other ribosomal proteins in the coexpression networks.

To identify novel components of protein synthesis with high confidence, we set to identify cutoffs that result in most accurate predictions of ribosomal proteins. The two parameters we investigated are (i) PCC cutoff required to identify co-expressed genes and (ii) the minimum percentage of ribosomal protein neighbors $(n)$ 

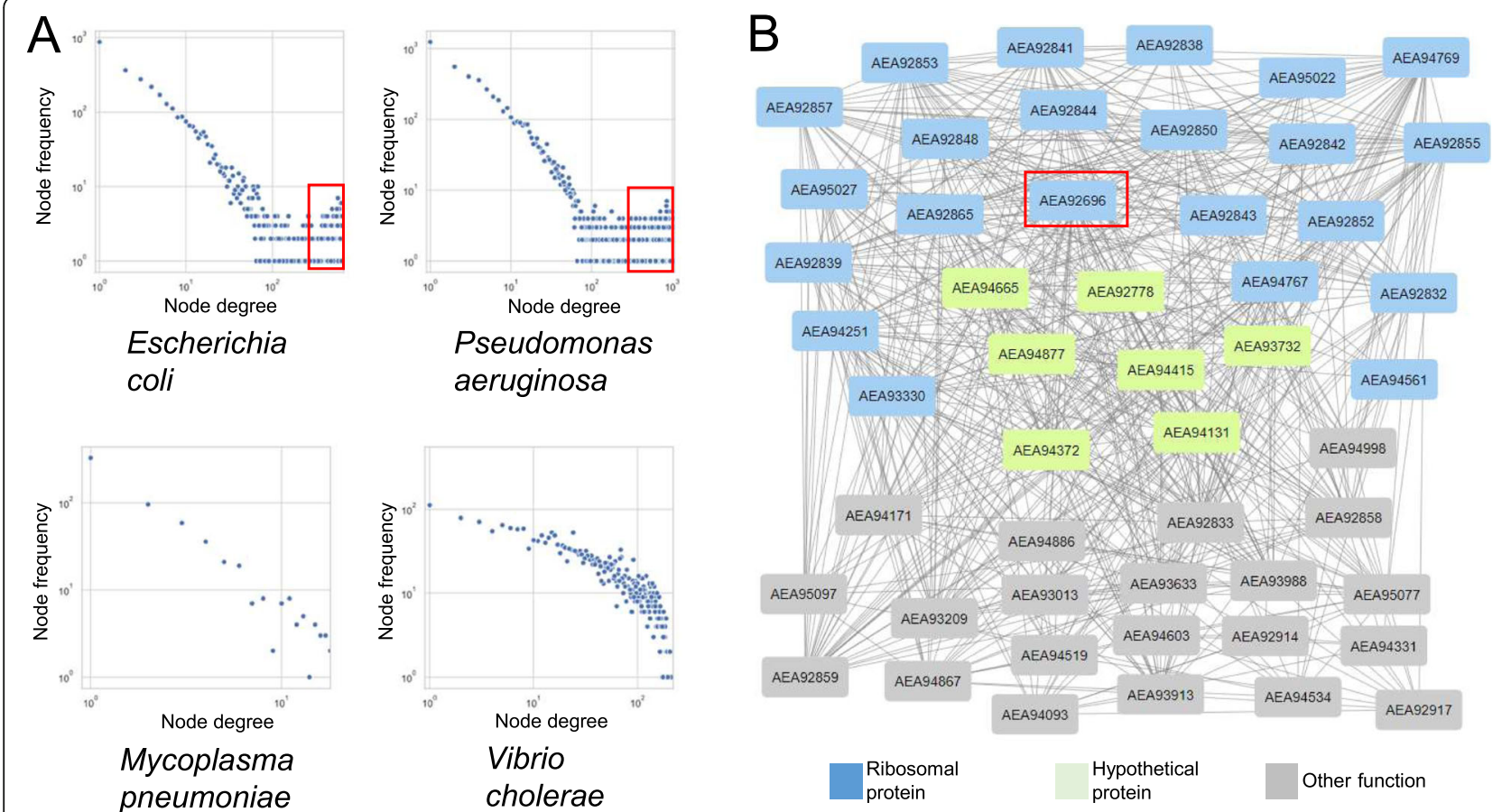

protein

Other function

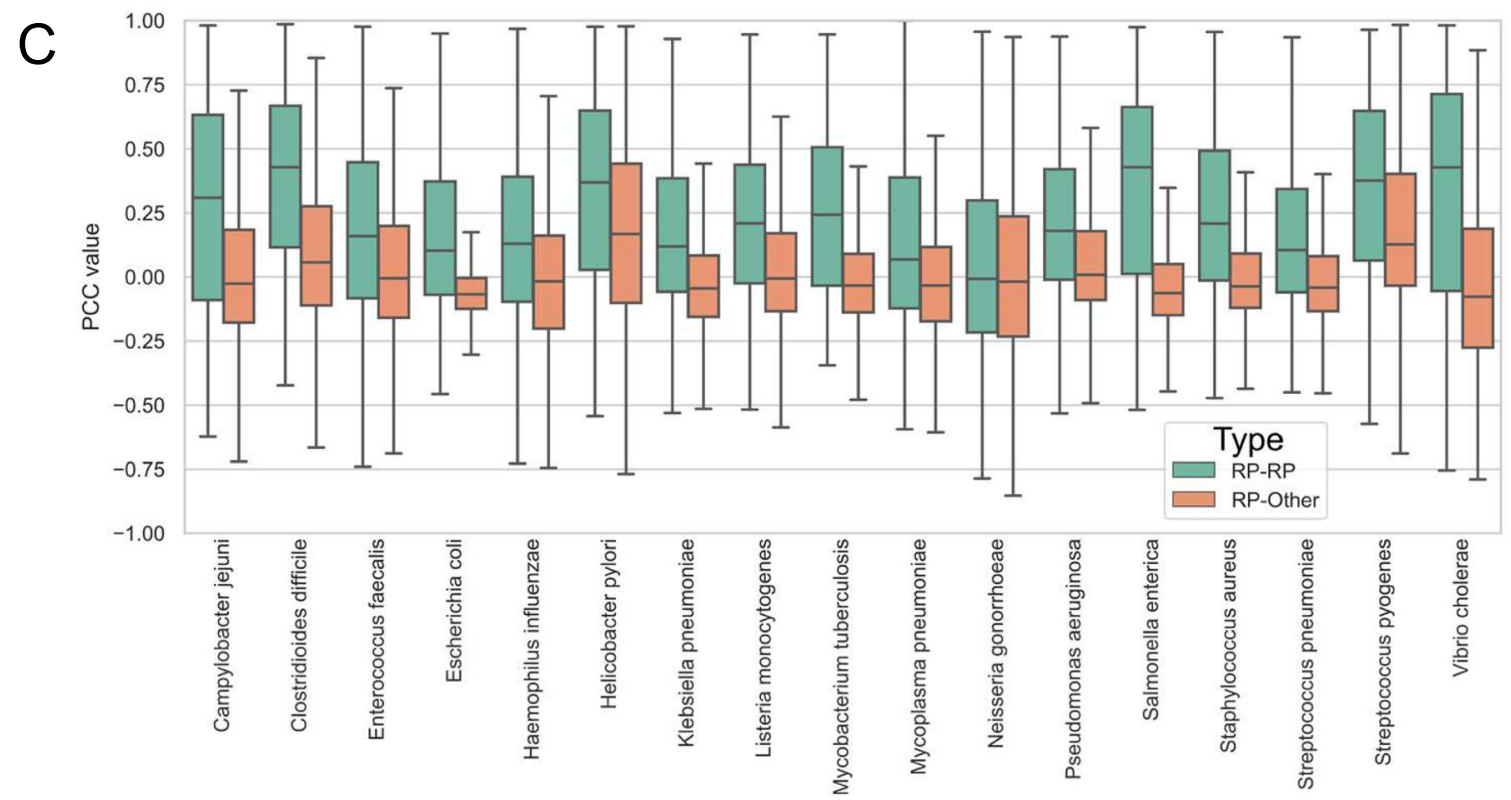

Fig. 3 Power-law and example of a ribosomal network. a Power-law plot obtained from the expression data of the 17 bacteria. The $x$-axis shows the node degree (number of co-expression connections of a gene), while the $y$-axis indicates the frequency of a degree. Pearson correlation coefficient (PCC) $>0.7$ was used to decide whether two genes are co-expressed. The two axes are log 10-transformed. b Co-expression neighborhood of AEA92696 from Enterococcus faecalis (red square), a 305 ribosomal protein S18, and the 50 most highly co-expressed genes (including AEA92696). Nodes indicate genes, while gray edges connect genes with PCC $>0.7$. Blue nodes represent ribosomal genes, green nodes represent genes with "Hypothetical protein" in their description, while gray nodes indicate genes with other functions. c Distribution of PCC values between ribosomal proteins (RP-RP, green bar) and ribosomal proteins and other genes (RP-Other) in the 17 bacteria. The $x$-axis indicates bacteria, while the $y$-axis indicates the PCC values 
required to assign a gene to protein synthesis. To benchmark the performance of the networks at these different cutoffs, each known ribosomal protein was treated as a gene with unknown function, and the ability of the networks to correctly predict the involvement of the ribosomal proteins in protein synthesis was scored. To score the performance, we used F1 score, which is a harmonic mean between precision (true positives/(true positives + false positives)) and recall (true positives/(true positives + false negatives)), where higher F1 score indicates high classification accuracy.

The analysis revealed the PCC cutoff and $n$ cutoff combinations that produce the highest $\mathrm{F} 1$ score for the $17 \mathrm{bac}-$ teria (Fig. 4a). The PCC cutoffs range from $>0.4$ (E. coli) to $>0.8$ (e.g., S. pyogenes), while $n$ values range from $20 \%$ (i.e., $>20 \%$ of co-expressed genes should be ribosomal proteins to assign a gene to protein synthesis) to $50 \%$. The F1 score was typically poor at low (0.1) and high (1) PCC cutoffs, as the networks likely connected too many irrelevant (for $\mathrm{PCC}>0.1$ ) and no relevant $(\mathrm{PCC}=1)$ genes. Similarly, the $n$ value typically resulted in poor F1 score at low (10\%) and high (100\%) cutoffs, as too many irrelevant genes $(10 \%)$ or too few relevant genes $(100 \%)$ were predicted to be involved in protein synthesis. Overall, the maximum F1 scores ranged from $\sim 0.4$ (Neisseria gonorrhoeae) to $\sim 0.7$ (Salmonella enterica).

We compared the performance of our co-expression networks to STRING network database (https://string-db.org/) [56], which integrates genomic neighborhood, gene fusion, genomic co-occurrence, co-expression, experimentally verified function, article text mining, and homology transfer. The comparison revealed that STRING performs similarly to our E. coli co-expression networks (Additional file 22: Figure S3, Fig. 4b), while for other bacteria, STRING showed higher F1 scores. This is not surprising, as methods that integrate multiple functional evidences tend to perform better than predictions based on only one evidence, such as coexpression [24, 27]. However, we note that only 4 (C. jejuni, E. coli, $P$. aeruginosa, and $S$. aureus) out of the 17 bacteria that we used in our analysis contained co-expression networks in STRING, which precludes STRING from using coexpression to identify genes involved in protein synthesis.

\section{Prediction of novel components of ribosomes by a meta- analysis of the co-expression networks}

To predict which genes with unknown function are involved in protein synthesis in the 17 bacteria, we first identified genes that are involved in protein synthesis (search term "ribosom") or shared no similarity to any characterized gene (search term "hypothetical," "DUF," "conserved"). The analysis revealed that typically, the ribosomal genes constitute $<5 \%$ of all genes in a bacterial genome (Fig. 5a). In comparison, the number of genes that are without functional annotation varies from
$<1 \%$ (Salmonella enterica) to 43\% (Helicobacter pylori) (Fig. 5b). Furthermore, between 7\% (Escherichia coli) and 23\% (Mycobacterium tuberculosis) of genes are orphans that do not belong to a gene family (Fig. 5c). Typically, genes with unknown function (orange bars) contain less Pfam domains than characterized genes (Fig. 5d, blue bars), but are frequently found in gene families (Fig. 5e, typically $>50 \%$ of genes, orange bars).

To predict uncharacterized genes that are involved in protein synthesis, we applied the same approach that we used to calculate the performance of the networks at different PCC and $n$ thresholds. More specifically, for each uncharacterized gene, we calculated the percentage of ribosomal gene neighbors $(n)$ at a given PCC cutoff (Additional file 23: Table S20). Since we have calculated the F1 score at the different PCC and $n$ thresholds, we could predict which genes are involved in protein synthesis at different $(>0.4,>0.5,>0.6)$ F1 score thresholds. By increasing the F1 score threshold, the prediction can be made more stringent, at the cost of the number of genes with the unknown function assigned to protein synthesis (Fig. 5f, Additional file 24: Table S21).

We observed a varying number of predictions between the different bacteria, ranging from 0 uncharacterized genes assigned to protein synthesis (Salmonella enterica) to 77 (Mycobacterium tuberculosis, Fig. 5f). As expected, the number of predictions dropped when the F1 score threshold was increased, with few genes assigned to protein synthesis at $F 1 \geq 0.6$ threshold. Interestingly, we observed a good agreement between the numbers of predictions made by different student groups. For example, Mycobacterium tuberculosis expression data (6495 samples, Table 1) was divided among 11 student groups and used to perform 11 independent predictions (group 1-11), which we compared to a prediction based on the combined data (all). The prediction based on all data (10 uncharacterized genes assigned to protein synthesis at F1 $>0.5$ threshold) did not contain more predicted genes than a subset of the data (e.g., 20 genes at $\mathrm{F} 1>0.5$, for group 11, 600 samples), indicating that more expression data does not result in more predictions. Furthermore, while each group predicted some unique genes, the majority of the predictions identified the same set of genes (Additional file 24: Table S21), indicating that more data is not necessarily better.

The 17 bacteria showed contrasting protein domain (Additional file 25: Figure S4A) and gene family (Additional file 25: Figure S4B) patterns for the predicted genes. For example, while $100 \%$ of the genes belonged to gene families or contained Pfam domains in E. coli, this was true for less than $40 \%$ of genes in C. jejuni (Additional file 25: Figure S4A). Furthermore, STRING analysis of the $11 \mathrm{E}$. coli genes predicted to be involved in protein synthesis (Fig. 5 f, all data, $F>0.6$ ), revealing that 

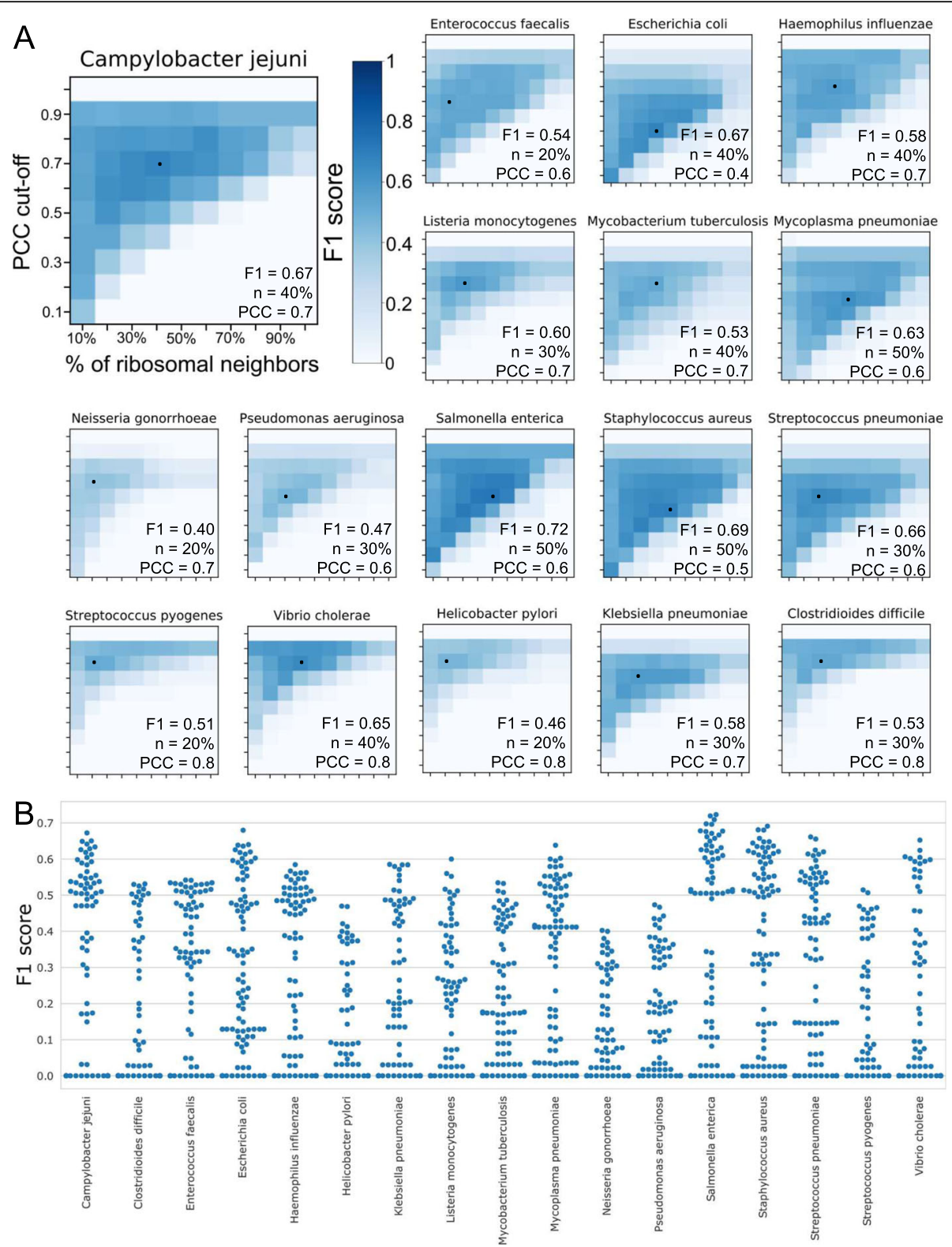

Fig. 4 Analysis of thresholds used to predict proteins involved in protein synthesis. a The heatmaps indicate the F1 score (harmonic mean of precision and recall) as a function of the percentage of ribosomal protein neighbors ( $n$ value, $x$-axis) and PCC value ( $y$-axis), for the 17 bacteria. The shade of cells in the heatmap indicate low (white) or high (dark blue) F1 score. The highest F1 score for each bacteria is indicated by a black dot, and the percentage of ribosomal protein neighbors ( $n$ ) and PCC value (PCC) are indicated in the lower-right corner of the heatmap. $\mathbf{b}$ Distribution of the F1 scores for the 17 bacteria. Each dot in the swarmplot indicates an F1 score obtained at PCC values ranging from 0.1 to 1 , and $n$ values ranging from 10 to $100 \%$

4 of the 11 genes could be associated with protein synthesis by STRING (Additional file 26: Figure S5). Conversely, the other 7 could not be associated with any function by STRING, suggesting that our approach is a valuable addition in gene function prediction in bacteria.

\section{Discussion}

Protein sequence similarity is commonly used to transfer molecular function annotation from one protein to another [57]. Molecular function annotation by sequence comparison is commonly performed using programs such as BLAST [58] and InterProScan [59]. However, a substantial proportion of coding sequences lack sequence similarity to any characterized genes (Fig. 4) [24, 36], making sequence similarity-based inference of gene function unsuitable. An excellent example of this limitation are genes that we have analyzed in this study. Since these genes are annotated as "hypothetical protein," "domain of 


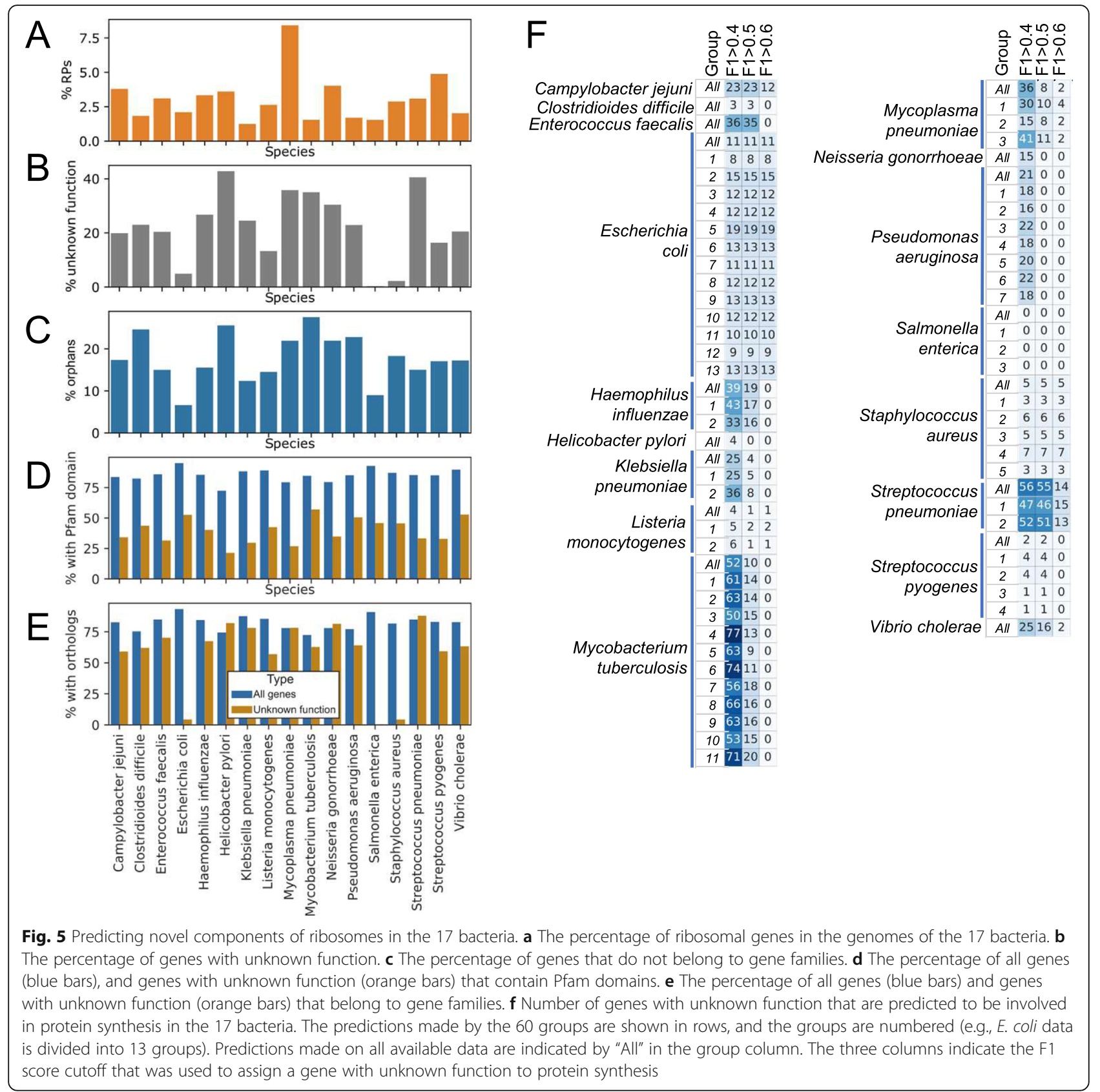

unknown function," or "conserved protein," they are likely not to share sequence similarity to characterized proteins.

Transcriptomic data is a rapidly growing resource that captures gene expression levels of all genes in an organism. Co-expression analysis is based on the observation that functionally related genes tend to have similar expression profiles across different experiments, and has become a powerful tool for predicting gene function [60]. We applied this approach to identify novel components of protein synthesis machinery in the 17 most notorious bacteria pathogens, for which sufficient (defined as > 100 RNA-seq samples) expression data exists (Table 1). In this study, we achieved two aims.

Firstly, we show co-expression analysis can be used to predict novel candidates of bacterial ribosomes. We observed that ribosomal proteins tend to be strongly coexpressed (Fig. 3b, c), suggesting that uncharacterized genes co-expressed with the ribosomal proteins are likely involved in some aspect (ribosome assembly, protein synthesis, termination) of protein synthesis. We predicted a substantial number of novel genes involved in protein synthesis for 16 out of 17 bacteria (Fig. 5f, Additional file 24: 
Table S21) that can serve as good targets to develop species-specific antibiotics. The available expression data for the 17 bacteria can be further mined to study other biological functions and vulnerabilities (e.g., cell wall, RNA, and DNA biosynthesis) of these bacteria.

Secondly, we show that such analysis can be outsourced to a large group of individuals. Here, the gene expression data was streamed and pseudo-aligned by 285 first-year undergraduate students, as part of the Computational Thinking class project. To this end, we used a modified LSTrAPCloud pipeline [47], where the students were divided into 60 groups, and each group was tasked to download and perform quality-control of $\sim 600$ samples over a week (Fig. 1 and 2, Additional file 1: Table S1). Theoretically, 85,500 $(300 \times 285)$ RNA-seq samples equivalent to $\sim 85 \mathrm{~Tb}$ could be processed per day by the class, providing a computing power rivaling a high-end computer cluster. While each student had access to only two Xeon cores, one of the major bottlenecks in processing the voluminous RNA-seq data, data download, was circumvented by fast Internet connection of each Google Colab virtual machine. Since each student uses a different virtual machine with an independent Internet connection, our approach demonstrates how a large quantity of data can be analyzed for free.

While similar approaches are used by, e.g., folding@home, it is to our knowledge the first attempt to process gene expression data in such a manner. We envision that similar approaches will soon allow us to study gene expression data within and across whole kingdoms of life.

\section{Conclusions}

To help identify novel components of bacterial ribosomes, we have used co-expression analysis to associate genes with unknown function to ribosomal proteins in 17 pathogenic bacteria. Our analysis identified more than 200 candidates for further functional studies, while our approach exemplified how such analysis can be outsourced to, e.g., a group of undergraduate students.

\section{Methods}

\section{Streaming RNA sequencing data}

The LSTrAP-Crowd pipeline was implemented on Google Colaboratory and is based on the LSTrAP-Cloud pipeline with standard parameters [47]. The pipeline streams the RNA-seq fastq files to a virtual machine in the cloud and deposits the processed gene expression data on the user's Google Drive. The CDSs were obtained from EnsembleGenomes and used to generate an index file by Kallisto [50], for subsequent estimation of gene expression. The RNA sequencing data of the 17 bacteria was obtained from European Nucleotide Archive (ENA) and mapped against the kallisto index of coding sequences (CDS) of the 17 bacteria. The used CDSs are Campylobacter jejuni (Campylobacter_jejuni_subsp_ jejuni_cg8421.ASM17179v2.cds.all.fa), Clostridioides difficile
(Clostridioides_difficile_e25.E25.cds.all.fa), Enterococcus faecalis (Enterococcus_faecalis_og1rf.ASM17257v2.cds.all.fa), Escherichia coli (Escherichia_coli_str_k_12_substr_ mg1655.ASM584v2.cds.all.fa), Haemophilus influenzae (Haemophilus_influenzae_r3021.ASM16975v1.cds.all.fa), Helicobacter pylori (Helicobacter_pylori_b8.ASM19675v1.cds.all.fa), Klebsiella pneumoniae (Klebsiella_pneumoniae jm45.ASM44540v1.cds.all.fa), Listeria monocytogenes (Listeria_monocytogenes_gca_001027125.ASM102712v1.cd-

s.all.fa), Mycobacterium tuberculosis (Mycobacterium tuberculosis_h37rv.ASM19595v2.cds.all.fa), Mycoplasma pneumoniae (Mycoplasma_pneumoniae_fh.ASM14394v1.cds.all.fa), Neisseria gonorrhoeae (Neisseria_gonorrhoeae_gca 001047275.ASM104727v1.cds.all.fa), Pseudomonas aeruginosa (Pseudomonas_aeruginosa_gca_001181725.E11_ London_26_VIM_2_06_13.cds.all.fa), Salmonella enterica (Salmonella_enterica_subsp_enterica_serovar_typhimurium_ str_lt2.ASM694v2.cds.all.fa), Staphylococcus aureus (Staphylococcus_aureus_gca_001212685.7738_4_

69.cds.all.fa), Streptococcus pneumoniae (Streptococcus_ pneumoniae_r6.ASM704v1.cds.all.fa), Streptococcus pyogenes (Streptococcus_pyogenes_ns88_2.SPNS88.2.cds.all.fa), and Vibrio cholerae (Vibrio_cholerae_v51.ASM15246v2.cds.all.fa). All available RNA-sequencing data for the 17 bacteria, comprising different growth conditions, media compositions and mutant strains, were downloaded and subjected to quality control. A total of 26,269 experiments were streamed (Additional file 1: Table S1), where each student used their own Gmail account to connect to their own cloud virtual machine (VM) provided by Google. To coordinate the download effort among the 285 students, the students mounted a Google Drive provided by the instructor. The drive was used to store all of the downloaded data. The main disadvantage of Google Colab VM is that each VM is stopped and erased by Google after 12 h. However, since the processed data is stored in a persistent manner on the shared Google Drive, the interrupted downloads can be easily resumed.

\section{Generating gene expression matrices for the 17 bacteria}

To remove RNA-seq samples that are of lower quality, we identified outlier samples that show a lower number (n_pseudoaligned) and percentage (p_pseudoaligned) of reads aligned to the coding sequences than the majority of the samples. This analysis assumes that the majority of samples are of good quality. The expression matrices containing the gene expression data that passed these thresholds are available in Additional file 3: Table S2, Additional file 4: Table S3, Additional file 5: Table S4, Additional file 6: Table S5, Additional file 7: Table S6, Additional file 8: Table S7, Additional file 9: Table S8, Additional file 10: Table S9, Additional file 11: Table S10, Additional file 12: Table S11, Additional file 13: Table S12, Additional file 14: Table S13, Additional file 
15: Table S14, Additional file 16: Table S15, Additional file 17: Table S16, Additional file 18: Table S17, and Additional file 19: Table S18. Additional file 1: Table S1 contains the n_pseudoaligned and p_pseudoaligned numbers and indicates which samples passed the thresholds.

\section{Identification of genes involved in ribosome biogenesis with co-expression networks}

To identify genes that are involved in protein synthesis in the 17 bacteria, we have first retrieved all genes containing "DUF" (domain of unknown function), "hypothetical," or "conserved" in their description. Next, we calculated the Pearson correlation coefficient (PCC) between the uncharacterized genes and all genes in the genome, where PCC thresholds ranging from 0.1 to 1 were used to indicate co-expressed genes. Finally, the uncharacterized genes were predicted to be involved in protein synthesis if $>10 \%,>20 \%,>30 \%,>40 \%,>50 \%$, $>60 \%,>70 \%,>80 \%$ or $>90 \%$ of the genes were coexpressed with contained annotations such as "ribosome" or "ribosomal."

\section{Identification of protein domains and gene families}

The protein sequences were obtained from EnsembleGenomes. We used Interproscan-5.44-79 [61] to obtain the Pfam domains. Groups of orthologous genes (gene families) were obtained using Orthofinder v2.3.12 [62] with Diamond [63], with default settings.

\section{Supplementary information}

Supplementary information accompanies this paper at https://doi.org/10. 1186/s12915-020-00846-9.

Additional file 1 : Table S1. Quality control of the RNA-seq samples. The table indicates the species (first column), sample ID (second column), group ID processing the sample (third column), number of pseudoaligned reads (fourth column), percentage of pseudoaligned reads (fifth column) and an indication whether the sample passed the set quality thresholds (sixth column).

Additional file $\mathbf{2}$ : Figure S1. Scatter plot showing the number ( $x$-axis) and percentage ( $y$-axis) of pseudoaligned reads for the 17 bacteria.

Additional file $\mathbf{3}$ : Table S2. Campylobacter jejuni expression matrix. Genes are found in rows, while samples are found in columns.

Additional file 4 : Table S3. Clostridioides difficile expression matrix. Genes are found in rows, while samples are found in columns.

Additional file $\mathbf{5}$ : Table S4. Enterococcus faecalis expression matrix. Genes are found in rows, while samples are found in columns.

Additional file $\mathbf{6}$ : Table S5. Escherichia coli expression matrix. Genes are found in rows, while samples are found in columns.

Additional file $\mathbf{7}$ : Table $\mathbf{S 6}$. Haemophilus influenzae expression matrix Genes are found in rows, while samples are found in columns.

Additional file $\mathbf{8}$ : Table S7. Helicobacter pylori expression matrix. Genes are found in rows, while samples are found in columns.

Additional file $\mathbf{9}$ : Table S8. Klebsiella pneumoniae expression matrix. Genes are found in rows, while samples are found in columns.

Additional file 10 : Table S9. Listeria monocytogenes expression matrix. Genes are found in rows, while samples are found in columns.
Additional file 11 : Table S10. Mycobacterium tuberculosis expression matrix. Genes are found in rows, while samples are found in columns.

Additional file 12 : Table S11. Mycoplasma pneumoniae expression matrix. Genes are found in rows, while samples are found in columns.

Additional file 13 : Table S12. Neisseria gonorrhoeae expression matrix. Genes are found in rows, while samples are found in columns. Additional file 14 : Table S13. Pseudomonas aeruginosa expression matrix. Genes are found in rows, while samples are found in columns.

Additional file 15 : Table S14. Salmonella enterica expression matrix. Genes are found in rows, while samples are found in columns.

Additional file $\mathbf{1 6}$ : Table S15. Staphylococcus aureus expression matrix. Genes are found in rows, while samples are found in columns.

Additional file 17 : Table $\mathbf{S 1 6}$. Streptococcus pneumoniae expression matrix. Genes are found in rows, while samples are found in columns.

Additional file 18 : Table S17. Streptococcus pyogenes expression matrix. Genes are found in rows, while samples are found in columns.

Additional file 19 : Table S18. Vibrio cholerae expression matrix. Genes are found in rows, while samples are found in columns.

Additional file 20 : Figure S2. Power-law plot of the 17 bacteria. The $x$-axis shows the node degree (number of coexpression connections of a gene, PCC > 0.7), while the $y$-axis indicates the frequency of a degree. The two axes are log10-transformed.

Additional file 21 : Table S19. Co-expression neighborhood of AEA92696 from Enterococcus faecalis. The genes are sorted according to the Pearson Correlation Coefficient ( $r$, first column). The gene IDs (second column), type (third column, 1 = ribosomal protein, 2 = gene with unknown function, $0=$ not 1 or 2 ) and annotation (fourth column) are indicated.

Additional file 22 : Figure S3. F1 score values for co-function networks obtained from STRING. Distribution of the F1 scores for 4 bacteria. Each dot in the swarmplot indicates an F1 score obtained at PCC values ranging from 0.1 to 1 , and $n$ values ranging from $10 \%$ to $100 \%$. Only the bacteria for which we could identify common gene identifiers in STRING and our analysis are included.

Additional file $\mathbf{2 3}$ : Table S20. Gene identifiers of uncharacterized genes predicted to be involved in protein synthesis. The columns indicate the gene ID (first), bacterial species and student group (second), PCC cutoff (third), number of ribosomal protein neighbors at a PCC cutoff (fourth) and number of all gene neighbors at PCC cutoff (fifth).

Additional file 24 : Table S21. Uncharacterized genes predicted to be involved in protein synthesis. Each row contains genes with unknown functions predicted to be involved in protein synthesis. The rows contain predictions made by each group (indicated by numbers) or by all available data (All data). The columns indicate the (i) bacteria, (ii) group ID, (iii-vii) predicted genes at the different F1 score thresholds.

Additional file $\mathbf{2 5}$ : Figure S4. Protein domain and gene family analysis of the genes predicted to be involved in protein synthesis. A) Percentage of genes with Pfam domains. B) Percentage of genes belonging to an orthogroup. The color bars indicate the F1 score threshold used to identify the genes.

Additional file 26 : Figure S5. STRING analysis of the 11 genes predicted to be involved in protein synthesis in E.coli by our analysis. The 11 genes are indicated with a red node. Below each network, functional enrichments that are detected by STRING are indicated. AAC75719, AAC74172, AAC75326 and AAC76626 are the four genes significantly associated with genes involved in protein synthesis.

\section{Acknowledgements}

We would like to thank Google for providing Google Colab and all members of the class of BS1009 (Introduction to Computational Thinking) that ran during the 2019/20 academic year. The students are Aaminatul Khalishah Binte Roslan, Adam Lee Xin Hong, Alena Tay Qi Ye, Ana Ho Sze Qi, Anastasia Griswold Thean Xue Ting, Andrea Tan Wenqi, Andrew Liew Yong Zhou, Ang Qian Qian, Ang Wei Ying, Ang Wen Hui, Anoushka Sameer, Aqidah Nafeesa Binte Mohamed Rafi, Ashlyn Ng Xuanqi, Aslam Firras Bin Azhar, Audrey 
Michelle Luminary, Beh Mei Zhen, Bernice Chan Hui Shan, Bertrand Wong Jern Han, Boon Kai Lun, Bridget Tang Jing Xing, Bryden Koh Yang Wei, Carissa Yuwono Kwantalalu, Celest Phang Lixuan, Chai Ruo Qi, Chan Mu En, Chan Tong Ling, Chan Yi Hue, Chang Jit Yee, Charlene Pek Jia Ning, Charmaine Chang Tze Hwee, Chee Mhin Chin, Chee Suhui Samantha, Chee Wei Heng Marcus, Chen Hui Xin, Cherie Hong Yi Xin, Cheryl Ann Ruthrayson, Cheryl Lim Jiayi, Cheryl Quek Chai Hui, Chia Rui Wen, Chim Ler Ting, Chiu Shun Xin Michelle, Chiu Wei Yeow, Chong Jia Shin, Chong Ming Jun, Chong Yu Hong, Chong Zia Yee, Choo Jialei Clement, Choo Jing Wen Germaine, Christie Tan Pei Ning, Chu Thi Sau, Chua Xin'er Cherlyn, Chua Yi Lin, Chua Yi Xuan, Clara Lie Kai Kee, Clare Yong Pei Yii, Clarice Keng Jing Ting, Clarice Lee Pei Xuan, Clarice Lee Zi Qi, Clarice Pohan, Daniella Bianca Cadayona Lim, David Wong Meng Kit, Devika Menon, Dhira Anindya Putri, Dian Atikah Bte Sharul Hisham, Dynn Sim, Edbert Edric Rodrigues, Edmund Tan Kok Hong, Er Kian Ching Gabbie, Esteban Ira Patricia Colendrino, Fan Chongyue, Fikri Bin Mohamed, Fong Tuck Choy, Foo Yan Xi, Foo Yong He Herman, Foong Wai Teng Melissa, Gabay Karen Dianne Aventurado, Genevieve Ling Tek Ting, Goh Wei Xuan, Grace Deng Zheyun, Hee Ker Min, Ho Abigail, Ho Jun Sheng, Ho Li Sheng, Ho Yan Ying, Ho Yu Hui, Ho Yun Ye, Hoh Ching Kwee, Hong Davin, Hoon Su Teng Megan, Hui Wai Hann, Ifa Syafiqah Binte Sulaiman, Jace Koh, Jaslyn Muk Cui Jin, Jazz Er, Jeraldine Poh Jie Min, Jes Kwek Hui Min, Jessica Lee Ru Xuan, Joe Ng Ren Guang, Jolene Lim Jiajin, Joseph Ng Jun Quan, Jovi Tan Siying, Joyce Wang, Ketti Boo Wenting, Khoo Jia Hui Olivia, Kirthana D/o Subramaniam, Koh Bi Qi, Koh Hui Fen, Koh Jing Han, Koh Shao Ning, Koh Ting Sween Kenny, Kong Jian Hua, Koo Wei Ling, Kuah Xuan Ye Chanel, Kwok Zi Rou, Lai Si Jia, Lai Yi Ni, Lam Zhi Sheng, Lee Heng Gei, Lee Ming Jern Adrian, Lee Thong Shuen, Lee Wan Xuan Trena, Lee Wei Jin Amanda Crystal, Lee Xin Yi, Leo Zhenn Yi, Leow Chen Yen, Lian Ying, Liauw Yong Tong, Liew Jia Hui, Lim Chuan Yu, Lim Dao Liang, Lim Jia Hui, Lim Jia Jia, Lim Jiaqi Alyssa, Lim Kai Peng, Lim Pei Xuan, Lim Qin Rui, Lim Shan Chun, Lim Shi Min, Lim Shing Yee, Lim Shu Hua Samantha, Lim Ting Yu, Lim Ye Joon, Lim Ying Xuan, Lim Yu Xian, Lim Yuen Shan, Lim Zi Jian, Lin Ming, Ling Zixun, Loh Dan Hong, Low Ann Don, Lui Ke Xin, M K Abdul Rahim, Mahathir Bin Mohamad Malaysia, Manessa Nah Shue Ern, Mathana Raj S/o Thayalan, Matthew Chua Yong En, May Ho Si Min, Mohammad Alfiean Bin Mohd Afdzanawar, Mohammad Dannial Bin Masrun, Moo Jia Rong, Mulupuri Sriya, Natasha Cassandra Chee, Neo Qi Ying, Ng Chuan Yi, Ng Jun Hao, Ng Peiran, Ng Ping Hui, Ng Rui Xue, Ng Thut Seng, Ng Wan Zhen Janice, Ngiaw Jia Xin, Nguyen Le Uyen Nhi, Nguyen Ngoc Minh Truc, Nguyen Thanh Son, Ni Ying, Nicholas Lee Bo Wen, Nur Afiqah Binte Mohammad Rizal, Nur Fareena Binte Sulaiman, Ong Si Yi Shirley, Ong Sze Yan, Ong Wei Wen, Ong Xuan Xuan, Ong Zhen Ying, Ow Yeong Yook Kit, Park Jiwon, Paula-teresa Chua Hui Yee, Phua Tian Xin, Poh Jia'en, Poh Jing Wen, Poh Zheng Yan, Poon Tze Yong, Pravenah Ravi Chandran, Quek Fu Cheng, R Vishalini Val, Ravi Keerthana, Reanne Lim Xi, Rebecca Liew Hui Ting, Rena Ho Si Hui, Rinta Pullamkottu Reji, Rohan Shawn Sunil, Rugdee Ryan Chieh Feng, Ryanjit Singh Kairon, See Jing Yi Julia, See Qi Rui, Seetoh Wei Song, Selia Yang Si Hua, Seo Min Li Gilia, Seow Jia Xuan, Shaun Ang Qiao Rou, Shellia Oktavina, Sherianne Tan Yen Tze, Sho Yu Wei, Shum Kai Xin Samantha, Siobhan Khoo Zi Yee, Siti Namirah Binte Roslan, Siti Nordiana Bte Sukry, Soh Jin Chun, Soh Kai Xuan Webster, Soh Shao Min, Su Peixuan Natalie, Subramanian Arthi, T Niraj, T Pravin, Tan Jia Wei, Tan Jia Ying, Tan Jia Yu, Tan Jun Xiong, Tan Nikita, Tan Qi Lun, Tan Qing Rong, Tan Wei Heng, Tan Xin Yee, Tan Xin Yi, Tan Yong Kang, Tan You Sheng Justin, Tan Zhe Jun, Tan Zi Ting Corrina, Tay Jian Hua, Tay Yi Ting, Tee Chu Yi Evangeline, Teffarina Tay Hui Wen, Teng Zheng Kai, Teo Jing Lin, Teo Yong Ren Johanan, Teoh Shook Wei, Teresa Tan Mien, Tey Siew Choo, Thein Mwei Aung, Thng Yu Xuan, Toh Hong Wei, Tok Zhi Ning, Tommy Lee Lam, Tracy Lee Si Hui, Trena Chan Yun Ting, Tristy Abigayle Marta, Tsou Han, Valerie Tang Yan Tong, Valerie Teo Fang Wei, Vanessa Lorraine Chea Yuen Leng, Vanessa Lunardi, Victoria Toh Le Yi, Victoria Tricia Tang Jing Yi, Villanueva Erielle Marie Fajardo, Wang Jue Lynn, Wee Shi Yong Denise, Wong Mee Yin, Wong Min Jia, Wong Pei Wen, Wong Shi Ying, Wong Xanaz, Wong Zhi Cheng, Wynnifred Lorraine Lee Jia Hui, Xylon Lee Wei Rui, Yee You Xian, Yee Yue Ru, Yei Xi, Yieo Jean Yi, Yip Weiqin Rayna, Yusuf Chouthury Shaik Farid, Zachariah Seet Zhong En, Zachary Jude D'rozario, Zhang Shurui, Zhao Margaret Xuan, and Zubaidah Binte Dadlani.

\section{Declarations}

The datasets supporting the conclusions of this article are included within the article (and its additional files). The authors declare that they have no competing interests.

\section{Authors' contributions}

MM conceived the project, $\mathrm{BH}$ and MM performed the analysis, QWT and WG developed the data download pipeline, and JWXN coordinated the data download. All authors read and approved the final manuscript.

\section{Funding}

We would like to thank Nanyang Technological University, Start Up Grant (SUG), for funding.

\section{Availability of data and materials}

All data generated or analyzed during this study are included in this published article, its supplementary information files, and publicly available repositories. The RNA-seq data was downloaded from European Nucleotide Archive. The sample identifiers used in this study are found in Additional file 1: Table S1.

\section{Ethics approval and consent to participate}

Not applicable.

\section{Consent for publication}

Not applicable.

\section{Competing interests}

None.

Received: 21 April 2020 Accepted: 12 August 2020

Published online: 03 September 2020

\section{References}

1. Spellberg B, Blaser M, Guidos RJ, Boucher HW, Bradley JS, Eisenstein BI, et al. Combating antimicrobial resistance: policy recommendations to save lives. Clin Infect Dis. 2011;52(SUPPL. 5):S397-428.

2. Mah TFC, OToole GA. Mechanisms of biofilm resistance to antimicrobial agents. Trends Microbiol. 2001;9:34-9.

3. Wilson DN. Ribosome-targeting antibiotics and mechanisms of bacterial resistance. Nat Rev Microbiol. 2014;12:35-48.

4. Arenz S, Wilson DN. Bacterial protein synthesis as a target for antibiotic inhibition. Cold Spring Harb Perspect Med. 2016.

5. Dunkle JA, Xiong L, Mankin AS, Cate JHD. Structures of the Escherichia coli ribosome with antibiotics bound near the peptidyl transferase center explain spectra of drug action. Proc Natl Acad Sci U S A. 2010;6(9):a025361.

6. Dinos GP. The macrolide antibiotic renaissance. Br J Pharmacol. 2017; 174(18):2967-83.

7. Wilson DN. The A-Z of bacterial translation inhibitors. Crit Rev Biochem Mol Biol. 2009:44(6):393-433.

8. Liu M, Douthwaite S. Activity of the ketolide telithromycin is refractory to Erm monomethylation of bacterial rRNA. Antimicrob Agents Chemother. 2002;46(6):1629-33.

9. Lin J, Zhou D, Steitz TA, Polikanov YS, Gagnon MG. Ribosome-targeting antibiotics: modes of action, mechanisms of resistance, and implications for drug design. Annu Rev Biochem. 2018;87:451-78.

10. Vazquez-Laslop N, Thum C, Mankin AS. Molecular mechanism of drugdependent ribosome stalling. Mol Cell. 2008;30(2):190-202.

11. Golkar T, Zielinski M, Berghuis AM. Look and outlook on enzyme-mediated macrolide resistance. Front Microbiol. 2018;9:1942.

12. Sharkey LKR, O'Neill AJ. Antibiotic resistance $A B C-F$ proteins: bringing target protection into the limelight. ACS Infect Dis. 2018;4(3):239-46.

13. Murina V, Kasari M, Takada H, Hinnu M, Saha CK, Grimshaw JW, et al. ABCF ATPases involved in protein synthesis, Ribosome assembly and antibiotic resistance: structural and functional diversification across the tree of life. J Mol Biol. 2019;431(18):3568-90.

14. Kerr ID, Reynolds ED, Cove $\mathrm{JH} . \mathrm{ABC}$ proteins and antibiotic drug resistance: is it all about transport? Biochem Soc Trans. 2005;33(Pt 5):1000-2.

15. Ahmed T, Yin Z, Bhushan S. Cryo-EM structure of the large subunit of the spinach chloroplast ribosome. Sci Rep. 2016;6:35793.

16. Ahmed T, Shi J, Bhushan S. Unique localization of the plastid-specific ribosomal proteins in the chloroplast ribosome small subunit provides mechanistic insights into the chloroplastic translation. Nucleic Acids Res. 2017:45(14):8581-95.

17. Barandun J, Hunziker M, Vossbrinck CR, Klinge S. Evolutionary compaction and adaptation visualized by the structure of the dormant microsporidian ribosome. Nature Microbiol. 2019;4(11):1798-804. 
18. Bieri P, Leibundgut M, Saurer M, Boehringer D, Ban N. The complete structure of the chloroplast 705 ribosome in complex with translation factor pY. EMBO J. 201736(4):475-86.

19. Kushwaha AK, Bhushan S. Unique structural features of the Mycobacterium ribosome. Prog Biophys Mol Biol. 2020:152:15-24.

20. Eyal Z, Matzov D, Krupkin M, Wekselman I, Paukner S, Zimmerman E, et al. Structural insights into species-specific features of the ribosome from the pathogen Staphylococcus aureus. Proc Natl Acad Sci U S A. 2015;112(43): E5805-14.

21. Greber BJ, Ban N. Structure and function of the mitochondrial ribosome. Annu Rev Biochem. 2016:85:103-32.

22. Melnikov S, Ben-Shem A, Garreau De Loubresse N, Jenner L, Yusupova G, Yusupov M. One core, two shells: bacterial and eukaryotic ribosomes. Nature Struct Mol Biol. 2012;19(6):560-7.

23. Melnikov S, Manakongtreecheep K, Söll D. Revising the structural diversity of ribosomal proteins across the three domains of life. Mol Biol Evol. 2018; 35(7):1588-98

24. Rhee SY, Mutwil M. Towards revealing the functions of all genes in plants. Trends Plant Sci. 2014;19:212-21.

25. Proost S, Mutwil M. Tools of the trade: studying molecular networks in plants. Curr Opin Plant Biol. 2016;30:130-40.

26. Usadel B, Obayashi T, Mutwil M, Giorgi FM, Bassel GW, Tanimoto M, et al. Co-expression tools for plant biology: opportunities for hypothesis generation and caveats. Plant Cell Environ. 2009;32:1633-51.

27. Hansen BO, Meyer EH, Ferrari C, Vaid N, Movahedi S, Vandepoele K, et al. Ensemble gene function prediction database reveals genes important for complex I formation in Arabidopsis thaliana. New Phytol. 2018;217:1521-34.

28. Hansen BO, Vaid N, Musialak-Lange M, Janowski M, Mutwil M. Elucidating gene function and function evolution through comparison of co-expression networks of plants. Front Plant Sci. 2014:5:394.

29. Proost S, Mutwil M. Planet: comparative co-expression network analyses for plants. In: van Dijk ADJ, editor. Methods in molecular biology. New York: Springer New York; 2017. p. 213-27. https://doi.org/10.1007/978-1-4939-6658-5_12.

30. Mutwil M, Obro J, Willats WGT, Persson S. GeneCAT--novel webtools that combine BLAST and co-expression analyses. Nucleic Acids Res. 2008;36(Web Server issue):W320-6.

31. Mutwil M, Ruprecht C, Giorgi FM, Bringmann M, Usadel B, Persson S. Transcriptional wiring of cell wall-related genes in Arabidopsis. Mol Plant. 2009;2:1015-24

32. Mutwil M, Usadel B, Schütte M, Loraine A, Ebenhöh O, Persson S. Assembly of an interactive correlation network for the Arabidopsis genome using a novel Heuristic Clustering Algorithm. Plant Physiol. 2010;152:29-43.

33. Mutwil M, Klie S, Tohge T, Giorgi FM, Wilkins O, Campbell MM, et al. PlaNet: combined sequence and expression comparisons across plant networks derived from seven species. Plant Cell. 2011;23:895-910. https://doi.org/10. 1105/tpc.111.083667

34. Ruprecht C, Mendrinna A, Tohge T, Sampathkumar A, Klie S, Fernie AR, et al. Famnet: a framework to identify multiplied modules driving pathway expansion in plants. Plant Physiol. 2016;170:1878-94. https://doi.org/10. 1104/pp.15.01281.

35. Ferrari C, Proost S, Ruprecht C, Mutwil M. PhytoNet: comparative Coexpression network analyses across phytoplankton and land plants. Nucleic Acids Res. 2018:46:W76-83.

36. Ruprecht C, Proost S, Hernandez-Coronado M, Ortiz-Ramirez C, Lang D, Rensing SA, et al. Phylogenomic analysis of gene co-expression networks reveals the evolution of functional modules. Plant J. 2017;90:447-65.

37. Ng JWX, Tan QW, Ferrari C, Mutwil M. Diurnal.plant.tools: comparative transcriptomic and co-expression analyses of diurnal gene expression of the Archaeplastida Kingdom. Plant Cell Physiol. 2019;61(1):212-20.

38. Wen Tan Q, Mutwil M. Malaria.tools-comparative genomic and transcriptomic database for Plasmodium species. Nucleic Acids Res. 2019; 48(D1):D768-75.

39. Proost S, Mutwil M. CoNekT: an open-source framework for comparative genomic and transcriptomic network analyses. Nucleic Acids Res. 2018;46: W133-40. https://doi.org/10.1093/nar/gky336.

40. Ruprecht C, Mutwil M, Saxe F, Eder M, Nikoloski Z, Persson S. Large-scale coexpression approach to dissect secondary cell wall formation across plant species. Front Plant Sci. 2011;2:23

41. Takabayashi A, Ishikawa N, Obayashi T, Ishida S, Obokata J, Endo T, et al. Three novel subunits of Arabidopsis chloroplastic NAD(P)H dehydrogenase identified by bioinformatic and reverse genetic approaches. Plant J. 2009;57:207-19.
42. Takahashi N, Lammens T, Boudolf V, Maes S, Yoshizumi T, De Jaeger G, et al. The DNA replication checkpoint aids survival of plants deficient in the novel replisome factor ETG1. EMBO J. 2008;27:1840-51. https:/doi.org/10.1038/emboj.2008.107.

43. Stuart JM, Segal E, Koller D, Kim SK. A gene-coexpression network for global discovery of conserved genetic modules. Science. 2003;302:249-55. https:// doi.org/10.1126/science.1087447.

44. Yu H, Luscombe NM, Qian J, Gerstein M. Genomic analysis of gene expression relationships in transcriptional regulatory networks. Trends Genet. 2003;19:422-7.

45. Lee I, Ambaru B, Thakkar P, Marcotte EM, Rhee SY. Rational association of genes with traits using a genome-scale gene network for Arabidopsis thaliana. Nat Biotechnol. 2010;28:149-56. https://doi.org/10.1038/nbt.1603.

46. Jiménez-Gómez JM, Wallace AD, Maloof JN. Network analysis identifies ELF3 as a QTL for the shade avoidance response in arabidopsis. PLoS Genet. 2010;6(9).

47. Tan QW, Goh W, Mutwil M. LSTrAP-Cloud: a user-friendly cloud computing pipeline to infer co-functional and regulatory networks. Genes (Basel). 2020; 11(4):428.

48. Tan QW, Mutwil M. Inferring biosynthetic and gene regulatory networks from Artemisia annua RNA sequencing data on a credit card-sized ARM computer. Biochim Biophys acta Gene Regul Mech. 2019;1863(6):194429.

49. Sibout R, Proost S, Hansen BO, Vaid N, Giorgi FM, Ho-Yue-Kuang S, et al. Expression atlas and comparative coexpression network analyses reveal important genes involved in the formation of lignified cell wall in Brachypodium distachyon. New Phytol. 2017;215:1009-25.

50. Bray NL, Pimentel H, Melsted P, Pachter L. Near-optimal probabilistic RNAseq quantification. Nat Biotechnol. 2016;34:525-7.

51. Patro R, Duggal G, Love MI, Irizarry RA, Kingsford C. Salmon provides fast and bias-aware quantification of transcript expression. Nat Methods. 2017;14:417-9.

52. Leinonen $R$, Sugawara $H$, Shumway $M$. The sequence read archive. Nucleic Acids Res. 2011;39:D19-21.

53. Broido AD, Clauset A. Scale-free networks are rare. Nat Commun. 2019;10(1): 1017

54. Barabási A-L, Bonabeau E. Scale-free networks. Sci Am. 2003;288:60-9.

55. Barabási AL, Oltvai ZN. Network biology: understanding the cell's functional organization. Nat Rev Genet. 2004;5:101-13.

56. Szklarczyk D, Franceschini A, Wyder S, Forslund K, Heller D, Huerta-Cepas J, et al. STRING v10: protein-protein interaction networks, integrated over the tree of life. Nucleic Acids Res. 2015:43:D447-52.

57. Kulmanov M, Khan MA, Hoehndorf R. DeepGO: predicting protein functions from sequence and interactions using a deep ontology-aware classifier. Bioinformatics. 2018:34:660-8.

58. Altschul SF, Madden TL, Schäffer AA, Zhang J, Zhang Z, Miller W, et al. Gapped BLAST and PSI-BLAST: a new generation of protein database search programs. Nucleic Acids Res. 1997;25(17):3389-402.

59. Quevillon E, Silventoinen V, Pillai S, Harte N, Mulder N, Apweiler R, et al. InterProScan: protein domains identifier. Nucleic Acids Res. 2005;33 SUPPL. 2.

60. Wu LF, Hughes TR, Davierwala AP, Robinson MD, Stoughton R, Altschuler SJ. Large-scale prediction of Saccharomyces cerevisiae gene function using overlapping transcriptional clusters. Nat Genet. 2002;31:255-65. https://doi. org/10.1038/ng906.

61. Jones P, Binns D, Chang HY, Fraser M, Li W, McAnulla C, et al. InterProScan 5: genome-scale protein function classification. Bioinformatics. 2014;30(9): 1236-40.

62. Emms DM, Kelly S. OrthoFinder: solving fundamental biases in whole genome comparisons dramatically improves orthogroup inference accuracy. Genome Biol. 2015;16(1):157.

63. Buchfink B, Xie C, Huson DH. Fast and sensitive protein alignment using DIAMOND. Nat Methods. 2014;12:59-60. https://doi.org/10.1038/nmeth.3176.

\section{Publisher's Note}

Springer Nature remains neutral with regard to jurisdictional claims in published maps and institutional affiliations. 